\title{
Virtual colonization
}

Social and Management Sciences $14 / 2$ (2006) 17

web: http://www.pp.bme.hu/so

c Periodica Polytechnica 2006

RESEARCH ARTICLE
András Margitay-Becht / Dana R. Herrera

Received 2007-09-14

\begin{abstract}
The intersections of virtual worlds, imagined communities and "real life" are producing complex liminal spaces that social scientists are gradually surveying. Although their economic potential and impact has been examined in great detail, the extensive effect they have on international relations and trade is so far largely unmapped. The paper will discuss the various spaces where the existence of virtual societies change the way countries and societies interact with one another, detailing the effect on culture, division of labour and international trade. Can it truly be optimal for a low-income economy to specialize in the production of virtual goods? What impact would these interactions have on different sectors of the nation-state? Do virtual societies encourage virtual colonization?
\end{abstract}

\section{Keywords}

virtual worlds liminal spaces technoscapes international trade

\section{András Margitay-Becht}

Department of Economics, BME, 1111 Stoczek u. 2, Budapest, Hungary e-mail: margitay@gmail.com

\section{Dana R. Herrera}

Department of Anthropology, St. Mary's College California, 1928 St. Mary's Rd., Moraga, CA 94575, USA

e-mail: dherrera@stmarys-ca.edu

\section{Virtual worlds}

A decade ago Appadurai (1996 p. 33) [1] developed a paradigm for examining "dimensions of global cultural flows" that included

technoscapes: "the global configuration, also ever fluid, of technology and the fact that technology, both high and low, both mechanical and informational, now moves at high speeds across various kinds of previously impervious boundaries" [1], (p. 34)

financescapes: "the disposition of global capital [that] is now a more mysterious, rapid, and difficult landscape to follow than ever before, as currency markets, national stock exchanges, and commodity speculations move megamonies through national turnstiles at blinding speeds, with vast, absolute implications for small differences in percentage points and time units" [1], (p. 34).

Today, these "-scapes" converge to produce a synthetic reality (or, virtual world) that expands and yet completely redefines what Appadurai termed "imagined worlds... the multiple worlds that are constituted by the historically situated imaginations of persons and groups spread around the globe" (p. 33). Virtual worlds are increasing in popularity and population as more people find it entertaining, preferable, and in some cases, profitable, to enter into these unreal dimensions and spend vast amounts of time inhabiting that space. Based on fourteen years of participant observation by the authors, as well as extensive interviews with virtual citizens, this article demonstrates (after a short introduction to the virtual worlds and international trade) that the emergence and growing popularity of virtual realities have the chance of radically changing the way we think about trade and international cooperation. The globalism of virtual worlds can redefine the boundaries between countries and continents, societies and individuals, and allow for faster economic and cultural convergence 1

\footnotetext{
${ }^{1}$ It is not the aim of this article to discuss whether - or when - this is a positive thing. Cultural convergence lowers the cultural diversity of our population, yet makes it easier to fit in a multicultural society. Economic convergence might
} 


\subsection{Description}

Virtual worlds are computer-based simulated environments inhabited by individuals through avatars. The worlds themselves can be two or three-dimensional, the avatars can be text-based or graphic, and the content can be thematized (in case of games) or free. For example, in the case of the immensely popular Diablo 2 (developed by Blizzard Entertainment), an individual could assume the identity (avatar) of a Paladin, Assassin, Barbarian, Necromancer, Sorcerer, Amazon, or Druid each with its own individualized skills and specialization. Common attributes of these worlds also include:

they provide a different "reality" in the virtual space; the storyline of the Diablo 2 environment takes the user's character through a series of quests while battling demons and collecting items (armor, weapons, spells, potions) that are potentially useful. Other games such as Second Life develop an environment devoid of fantasy or science fiction elements, thus mimicking the Real World.

the avatars the subscribers use to access the virtual world inaccessible to them, are stored on the provider's servers; thus making hacking the avatars (and changing their possessions through techniques outside of the virtual world) impossible

the avatars are capable of communicating with each other; players therefore interact not only with the artificial intelligence of the computer game but with other gamers located anywhere in the world.

The third point illustrates why virtual worlds steadily increase in popularity. They were designed to be more entertaining and absorbing than reality - they had to be, otherwise few would leave the Real World for something virtual. Game developers quickly realized that synthetic worlds were the new a conduit for increased, intensive social interaction. Broadband Internet and better computer hardware made it possible to have more absorbing user experience, allowing dozens or even thousands of avatars to be actively engaged in communication. Well developed plot lines, eye-catching graphics, and a world that could be explored repeatedly heightened the stimulation of the imagination. Thus, despite time differences, distance factors, and potential sociocultural barriers, a Druid from the Philippines might interact with a United States Paladin and a Hungarian Necromancer as citizen-warriors of a virtual world that battle together against the forces of evil; or a British shoemaker could spend time with a South African bank manager in a virtual Jacuzzi in Second Life. Kelly [5] and Taylor [11] for example, explore how these international collaborations develop a metacommunity beyond the borders of traditionally recognized nation-states making gamers more immersed in the Virtual Worlds than before and in essence creating a reality that can and does become more significant to the user than the "Real World."

not appear as problematic at first light, but it is the primary drive behind cultural convergence.
There are no signs that the popularity of virtual worlds are on the decline. In reality, a near-exponential growth can be experienced, as each successive "generation" of virtual realities have a magnitude more players than the generation before. Ultima Online (1997) had about 240.000, EverQuest (1999) approximately 550.000, RuneScape (2002) 780.000, whereas World of Warcraft (2004) has over 8.500.000 players [http://www.mmogchart.com/Analysis.html], and the non-game Second Life surpassed 4.6 million subscribers [http://www.secondlife.com/whatis/economy_stats.php]. These increasing demographics of people also suggest that Appadurai's technocapes and financescapes converge in a way to produce liminal spaces between real life and synthetic worlds; rather than specific incidents being confined to either of the virtual or the real world, increasing socioeonomic relationships between inhabitants of both places stimulate the development of such phenomena such as Real Money Trading.

\subsection{Real Money Trading}

The increasing involvement in virtual realities gave rise to the phenomenon of Real Money Trading (RMT). This denotes the activity of trading goods produced (and present) in virtual communities for real money. For example, someone who has a warrior/hunter avatar in the GuildWars synthetic world might elect to sell some of her hard-earned in-game currency for real money. This is something to be expected, since the wealth of individuals might be different in the two commodities (real and virtual), so there is place for some trade there. RMT, however, means that it becomes possible to earn money playing a game/being engaged in a virtual world, thus the immersion to virtual existence becomes complete.

\section{International trade}

Trade is one of the oldest activity of humanity: willful exchange of goods perceived as near-equivalent value. Trade happens whenever two individuals perceive the value of two commodities differently: the moment they realize this different evaluation, they will swap. The reason for the different perceived value can come from unequal allocation (person $\mathrm{A}$ has a lot of the first good, whereas person B has a lot of the second good), or different preferences (person A might dislike one good, while person B might dislike another).

The simplest reasoning for international trade can be the different resource allocation: if one country has an abundance of the resources needed to produce the first good, whereas the other country can easily produce the second good, then trade will start 2

\footnotetext{
2 This theory, going against the then-mainstream mercantile thinking, was first fully developed by Adam Smith. He developed the idea of the exchangeable value, based on "the toil and trouble of acquiring" the good. $\llbracket 10$
} 


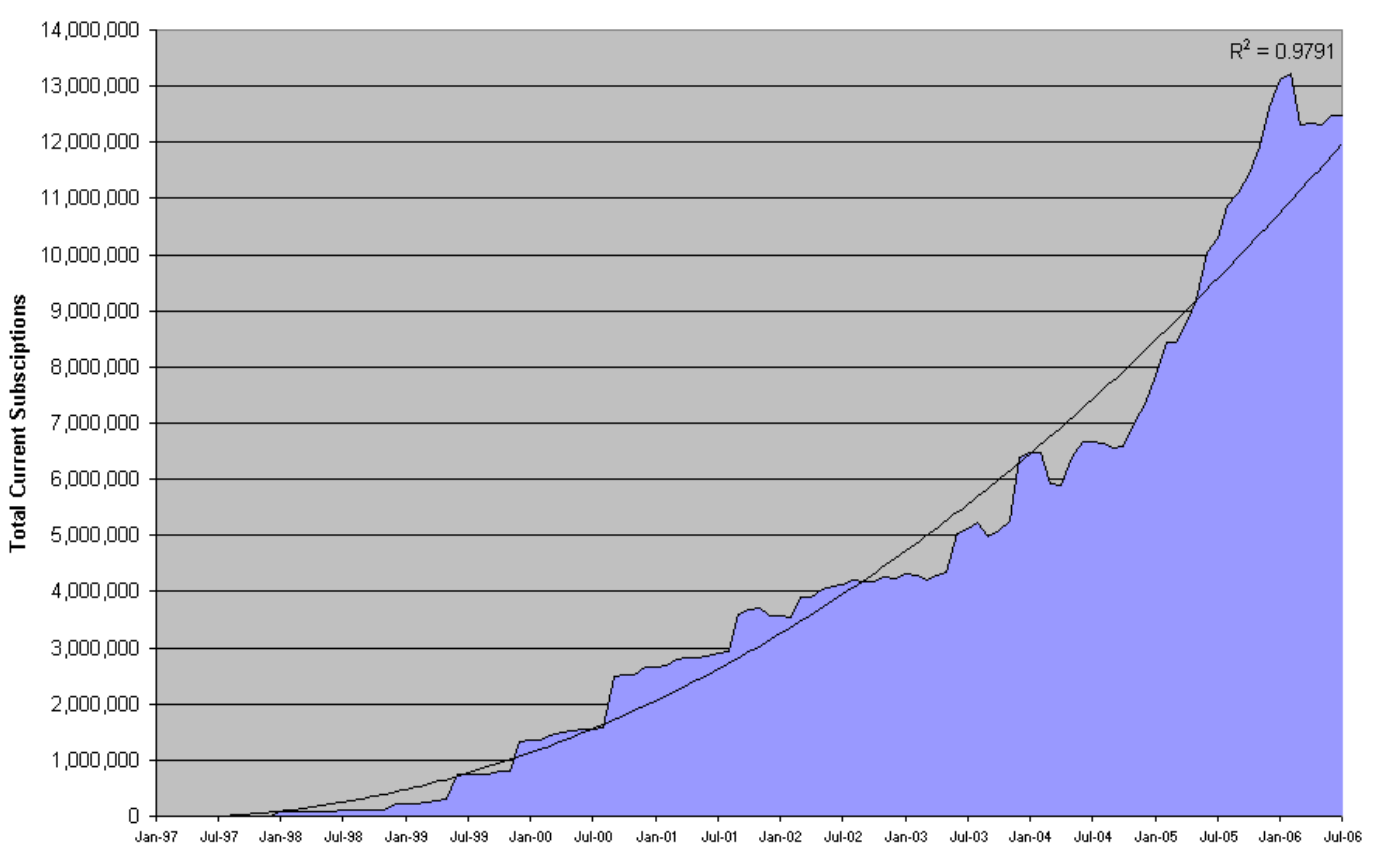

Fig. 1. Total number of subscribers. Source: mmogchart.com

\subsection{Comparative advantages}

The theory of comparative advantages, developed by Ricardd ${ }^{3}$ states, that international trade can arise, even if one nation is more productive and better equipped to produce both goods. In this case, the less efficient nation has a comparative advantage in that field, where it is least ineffective compared to the other nation. This forms the basis for colonization: even though the colonizer can produce everything more effectively (due to the greater technological advances at it is disposal), it is still worthwhile for it to move its low tech production out of the country, so that they can focus on the more sophisticated production tasks.

\subsection{Considerations}

Many economic models do not consider significant real-life factors when discussing international trade. The HecksherOhlin model (based on the Ricardian approach), for example, assumes that the production function is the same across regions (that is, the same kind of technology can be used everywhere around the globe). In reality, this could not be further from the truth: "human capital" is a determinant factor to the efficiency of production.

Some notable factors:

Human capital: the accumulated knowledge in the workforce regarding production technologies. A region rich in human

\footnotetext{
${ }^{3}$ Ricardo questioned the Smithian approach to value: "Adam Smith, [...] for the purpose of determining the varying value of other things, has himself [...] chosen a medium no less variable.". [9] He states, that instead of using absolute values, the proportionate labor usage is the determining factor between two goods.
}

capital can utilize the same production technology more effectively, thus generating greater output using the same amount of capital and labor, than a land scare in human capital.

Latent development: a combination of work ethics and mentality, employer-employee relations. There are large number of studies indicating that it is difficult to compare working times across regions. Even if one considers the quality of work to be the same, the measurement unit can hide different meaning. A study by the Swiss bank UBS, for example, revealed that there are huge differences between the working hours within a given week among nations: while the French worked an average of 35 hours, some areas of China faced 52 hour or longer workweeks [4]. Another study indicates, that even working hours are not an obviously good and universal measure across regions: as the annual Proudfoot Productivity Report shows, large amount of work time is going to waste, and this can vary as much as $11 \%$ of the total working hours across regions [8].

These factors have effects not only on efficiency and costs, but on the quality of the production as well. If we are to introduce the concept of different quality of goods, then we are not talking about the same good anymore: there are multiple production functions, one for each "quality" of the good there is. Some of these functions might not be usable for some nations, and the "shape" of these functions can be different among nations.

The moment we take these issues into consideration, international trade suddenly becomes significantly more complicated. In this case, the ability of various nations to provide "good" quality products becomes increasingly significant: higher quality goods can demand better exchange rates, and might force 
lower quality products out of circulation.

Another generally overlooked issue is the fact that not all goods are traded on the marketplace. This means that some part of a nation's production cannot be sold to others - and at the same time, some part of its consumption cannot be substituted with import. Considering international trade, this distinction divides the marketplace: there is the international marketplace of tradable goods, where both the local producers and local consumers trade, and there is the strictly national marketplace of non-tradable goods, where local producers sell their goods to local consumers. And while producing non-tradable goods can be rather profitable, it does not improve the nation's trade balance.

\section{International virtual trade}

The international virtual trade combines the real money trading with international trade: in essence the virtual goods are produced in one nation, and sold in another.

\subsection{Virtual trade vs. real trade}

The international virtual trade has a set of attributes which set it apart from standard trade. These effects have both theoretical relevance, and can be utilized to further profits and improve regions.

\subsubsection{Uniform quality}

Goods produced in a virtual world are by their very nature of uniform quality, since their parameters are determined by the laws of the synthetic environment. The production technology determines only the price and the scarcity of the created good, not it is quality or parameters. This allows, that all virtual goods be treated as equals, so the standard quantitative economic approach can be used in modelling their production and their market. When comparing with trading material items, however, this is a huge difference: no matter the source, the goods on the market are equal ${ }^{4}$

\subsubsection{Dual production technology}

As opposed to production in the real world, the production of virtual goods is done with two distinct set of capital: "real" capital and "virtual" capital. The peculiarity is that the "real" capital is near-identical: computer, program, Internet connection is all that is required for taking part in virtual production. One such set enables one instance of the virtual worker to be present

\footnotetext{
4 Throughout this article the assumption is, that virtual items are created through taking part in the virtual world, and not via the use of hacks. The presence of the so-called "dupes" or duplicates, which are copies of other existing virtual items, upsets this statement - and the virtual marketplace, too. For this reason, the service providers try to fight their propagation, and this, in some cases, result in two distinct kinds of virtual items: the stable, legitimately found ("legit") items, and the dupes, which get deleted periodically. This difference in quality, however, is not due to differing physical capital or inherent labour qualities, rather to the technology used, and most modern virtual worlds make such duplicating efforts near impossible.
}

in the synthetic world. "Virtual" capital is the kind of virtual equipment needed for producing the virtual goods within the virtual worlds (virtual swords and armor, for example). These goods can be purchased (either for money in Real Money Trading or for virtual money) or "produced" the same manner the final products will be produced.

The third factor of production is labor. Labor uses the real capital to enter the virtual world, and the accumulated virtual capital in there to produce the virtual goods to be sold later on. It is, however, not measured as easily as in real production: there are various ways in a virtual production scheme for a single physical laborer to have multiple avatars within the same world, cooperating with each other, thus greatly enhancing her/their productive potential. This, however, can fall under the category of capital, though multiple avatars require both extra physical and virtual capital.

\subsubsection{Human capital, know-how}

The effect of human capital is present even in virtual production: depending on the virtual reality, the in-world development methods might require various knowledge of the virtual place to be an efficient producer. This "knowledge" might be "virtual" or "real", depending on whether it concerns the virtual world itself, or the way the subscribers interact with the virtual worlds. Virtual knowledge is knowledge of the virtual world (what is the most effective way of producing a certain kind of equipment, or how does one generate most efficiently in-game currency), whereas real knowledge is knowledge of the real technologies used in virtual worlds (the client-server architecture, the interface to the clients, the communication protocols etc).

Real knowledge might be legal (for example, in case of World of Warcraft, it might be a set of add-on tools which enable better control over the world interface or allow for better coordination among workers), and illegal (similar tools used in Diablo 2, another game from the very same company but with a different end user policy). The operators of the virtual worlds determine the boundary between legal and illegal knowledge.

\subsection{Differences across worlds}

International virtual trade differs across the host synthetic worlds, too. There are some worlds which discourage it, but aside from legal actions, do nothing. Some worlds try to raise as many technological blocks in the way of RMT as possible (binding items to avatars, essentially making them non-tradable, for example). The other extreme approach is when a virtual world completely embraces real money trading.

\subsubsection{World policies}

The most important difference across worlds is the general approach to real money trading. It is notable, though, that RMT is present in nearly all popular synthetic worlds, even in the rather protective ones. This is mostly due to the currency phenomenon: every market will develop it is own currency. It has to be 
tradable, scarce enough to have value but frequent enough to be able to facilitate trade among the members. Because of the tradability restriction, this virtual commodity will always appear in $\mathrm{RMT}^{5}$

The situation is drastically different for those synthetic worlds which embrace real money trading. Second Life, for one example, based it's business model around RMT: it's own currency (the Linden dollar, L\$) is exchanged against the USD on it is own virtual marketplace. Everything in the world can be purchased for Linden dollars, thus it is value is unquestionable within the synthetic world. Second Life's owner and provider, Linden Labs, provide data for daily exchange rates and volumes of L\$ traded. Subscribers are welcome to generate content to the world, and they can sell off that content for $\mathrm{L} \$$, that can be freely exchanged to US\$ on the Linden dollar marketplace (LindeX).

Second Life also positioned itself in a way to emphasize the potential profits of real money trading. They not only publish information about the L\$ exchange rate (http://secondlife.com/whatis/economy-market.php), but also list economic activity within the world, focusing on money making and spending. As of March 14, over 25,000 subscribers were making money in Second Life, more than 600 of them over 1000 USD. On the other side, nearly 230,000 subscribers were spending it, and nearly 600 of them spent over 1M L\$ in February (at the time, 1M L\$ was roughly worth 3700 USD).

\subsubsection{Legal issues}

Considering that the virtual worlds are composed of computer programs and data stored on the servers of the providers, the ownership of virtual commodities is not trivial. Most providers keep ownership of all data on their servers, and only allow rightof-use to their subscribers. This means, that the avatars themselves and anything owned by them are the rightful possessions of the providers. Some providers use this ownership to file lawsuits against anyone involved in RMT, whereas others allow that practice.

The reaction to the providers' stance by the community is also a determining factor. Some believe, that RMT disrupts the "balance" of the virtual world, since those, who are not so involved with the given synthetic world can "buy their way in". Considering that the volume of real money trading was over one hundred million dollars even in 2005 [12] we can deduct, that sufficient number of subscribers find it acceptable and appealing to make it viable.

\subsubsection{Provider production}

The greatest danger to Real Money Trading: what happens if the providers of the virtual worlds engage in the production

\footnotetext{
5 The "currency" in each marketplace is not necessarily the intended money of the virtual world. In the Diablo 2 marketplace, for example, the currency of exchange is the "high rune", even though gold is the declared money in the game. The overabundance (and relative uselessness) of the in-game gold made it worthless, so traders settle their deals in runes.
}

of virtual goods? While not an issue to the commerce-based virtual worlds (like Second Life), this can seriously upset the balance of in-game trade in most synthetic worlds. In most cases providers are capable of producing at virtually no cost, thus are capable of undercutting the prices of the subscriber-producers 6 It is not only touchy because it has the potential of destroying Real Money Trading, it is also questionable since it runs the risk of disrupting "game balance" 7

Most virtual worlds heavily use scarcity as a drive for subscribers: they need to invest more time to be able to possess the particular virtual good. If this scarcity is "removed", then players might loose interest in the virtual world altogether.

\subsection{Effects on society}

Considering the peculiarities of production of virtual goods and their trade, a new degree of international division of labour emerges along the lines of average wage levels. The demand is formed of individuals with higher personal income, since they sacrifice more potential income per hour spent in a virtual world than individuals with lower personal income, who, in turn, become the suppliers. Since the average wage levels are different among nations, the low(er) income nations become the general suppliers of virtual goods, whereas the high(er) income nations provide the demand.

The usual setup is that members of the high income nation hire members of the low income nation for production purposes. This usually happens as a form of "investment": the workers get their monthly wages, some form of marketplace needs to be established for the virtual goods, a marketing campaign has to be set in motion (the "location" for that can be in both the real and virtual world) etc. Due to this need of investment, large RMT houses are usually located in the United States. Since in order to take part in the production process, the workers need a good IT infrastructure, but at the same time have to face low average personal income, the workers usually come from some East-Asian nation 8 (This, of course, is not always true; the trading house VSG is located in Hungary, using local students as workers.)

\footnotetext{
6 This is not true to Second Life, since RMT there involves labour-intensive production: either new virtual goods that require artistic and/or computing work, or social services, that require intense in-game presence.

7 From a purely economic standpoint, the providers have an option to become monopolist in the RMT scene. This option causes an interesting decision to be made: when should the company call it? It depends on the stability of the world (whether calling the option would destabilize the economy, this itself depending on how wide spread is RMT already, and how much resistance would the population raise against the company's decision to sell virtual goods), on the potential profits to be made by provider production, and on the potential loss caused by player disillusionment and migration.

${ }^{8}$ It is interesting to note, that although in June 2006 World of Warcraft had over 6.5 million subscribers, according to mmogchart.org at most 3 million of these lived in the United States or Europe. Most of the users are Chinese.
} 


\subsubsection{Colonization}

Because of the usual international division of production (the virtual workers are located in a low income nation, their employers and the actual market is located in a high income nation), virtual production parallels the production structure of the colonial times. It is expected, that this colonization will "shift" towards self-employment later on: when sufficient capital is accumulated in a low income nation, some local companies will be formed to sell their own products. This is relatively easy to do, since:

the goods are of uniform quality

the delivery of the product happens in the virtual world, thus there is no distance, no agglomeration no transportation costs the "real money" purchases usually happen over the Internet

the marketing targets either the virtual world itself, or focuses only on the Internet as a real-life appearance.

Current producers try to fight this tendency by merciless pricewars, lowering their own profit ratio to $10-20 \%$, thus trying to form a kind of "economic barrier to entry". This essentially prevents new entries, since the fixed cost of entering is hard to earn back at this point. This is due to the fact that the nearperfect competition drives down prices (this is doubly true to virtual worlds where there are relatively few goods available for sale), so RMT trading houses that did not enter the market early enough are essentially locked out ${ }^{9}$

Another barrier to entry is that of trust. The buyers are more willing to trust a web-site local to their place of residence than an off-shore site. This, however, is only another form of monetary barrier: all an off-shore trading house has to do is register a local domain. Payments are going through PayPal usually, so the only true barrier is their service: to which nations are they willing to provide payments. Even in regions where the producers can not directly enter the market, the colonies' dependence can reverse, or change to a co-dependence: the producers rely on the resellers for their better reputation (and infrastructure), whereas the resellers depend on the higher "human capital" accumulated by the producers (since they work out methods of "farming" virtual goods, and they gain experience by actually doing it).

\subsubsection{Sectoral Changes}

Considering that current producers of the RMT scene earn comparatively well, and that their work environment is comfortable and the work itself is not exhausting, while at the same time

\footnotetext{
${ }^{9}$ During our interview with VSG he mentioned, that in order enter the WoW market would cost him approximately 100,000 USD. He also claims, that due to the diverse nature of goods available for sale in Diablo II, he can keep his profit ratios relatively high - even though the game itself is rather old. In the much newer WoW the main tradable commodity is gold, thus it is relatively easy to compare prices. This drove down profit ratios, and that, coupled with the steep price of entering, keeps many smaller traders out of the WoW marketplace.
}

they are able to produce internationally tradable goods, it is to be expected that this sector is facing a boom. In reality, with the boom of the virtual worlds, RMT is facing a boom itself. The question arises: is it worthwhile for a nation to focus greatly on virtual production? There are a number of effects to drive a nation to shift its production towards virtual goods, causing sectoral changes:

High wages, better quality of life: individuals will shift towards virtual production (in Hungary, the virtual workers earn four times the minimum wage, and make approximately the same amount of money as the average salary of highly qualified labour, whereas the work itself requires little or no education)

High quality production, easily tradable goods: trade balance improves for the producer nation (the virtual goods are essentially exported: even though they always reside within the virtual world, the movement of payments indicate an export from the point of view of the producers)

Exponential expansion of virtual worlds mean an ever increasing market, which in turn means that it is worthwhile to focus greatly on virtual production, and help the development of this sector. For the nation this means, that development of a better information infrastructure (better Internet connection) can result in a huge improvement in the trade balance and export figures.

Considering that real money trading has beneficial macroeconomic effects, it could be important for a government to try to avoid virtual colonization. If the above described duality develops, then the owners of the trading houses, usually located in high income nations, reap the most of the profits from the virtual trade. By providing help to develop local production vertical corporations, that are large enough to be able to focus on wholesale and retail activities aside from the production itself, a government can ensure that larger percentage of the profits from real money trading remain within the country. Without this, the country essentially becomes a raw resource provider (in this case the raw resource is cheap labour).

\subsubsection{Taxation}

RMT, by its very nature, is invisible. Cash flows appear on the seller's account, but that account can be located anywhere even in a third country. As more and more people change their professions to be virtual producers, the tax income of the lowincome nation will drop drastically. This is doubly troublesome, since the increasing overall income of the nation couples with a decrease in state revenues, so the government is less able to provide the services it provided before - and at the same time, it might never regain the resources it invested in the development of the information infrastructure and/or local production verticals in the first place. 


\subsubsection{Dutch disease}

Dutch disease is the name of the sectoral shift to accommodate some change in the nation's external state. The nation splits along the lines of a "booming" and a "lagging" sector, and resources will be pooled in the booming sector for higher profits. The third sector of the nation, the non-tradable goods, will also experience a boom, since the booming tradable sector will bring in higher revenues to the nation, thus allowing for members of that sector to spend more resources on the non-tradable goods. Because of this, the "lagging" sector empties: some leave for the booming sector (direct de-industrialization, resource allocation effect), while others leave for the non-tradable sector (indirect de-industrialization, spending effect). The spending effect also increases the price of the goods in the non-tradable sector, which causes a real exchange rate appreciation (since prices in the tradable sectors are set internationally).

The real problem arises, when the booming sector collapses. If the boom lasted long enough, the lagging sector is severely weakened, thus the nation is left with a strong non-tradable sector and a rather weak (and probably uncompetitive) tradable sector, meaning that the nation will face a severe trade deficit.

In case of the virtual goods, some elements of Dutch disease can emerge. The booming virtual trade sector will cause a degree of direct de-industrialization, while the potentially higher income in the booming sector might cause some indirect deindustrialization. There are two variables which determine the extent of Dutch disease:

the actual boom: how fast do the virtual societies expand. If the current exponential rate remains, direct deindustrialization will be a significant effect most infrastructurally developed low income nations have to face

the extent of openness: if the low income nation is relatively open, then the size of the non-tradable sector is negligible, meaning that the indirect de-industrialization will not be a significant factor

This means, that depending on internal and external factors, the development of Dutch disease can differ greatly from nation to nation.

The greater danger of Dutch disease is the sudden disappearance of the originating force. If, for some reason, virtual realities would become obsolete, a large number of people would be without work suddenly, and the de-industrialized state of the low income nation could mean a severe lag in development and quality of life. However currently there are no signs to indicate such change.

\section{Conclusions}

Trading virtual goods results in real money. Real money trading enables subscribers to virtual worlds to exchange their time spent in the virtual reality to real money by producing virtual goods, and this has the potential of not only affecting the individual, but the nation, as well.
Focusing on the individual, real money trading allows for producing goods which can be sold internationally, in a quality identical to that produced by anyone else. There is little capital barrier to entry: with the equipment needed to join the virtual world, any subscriber can produce virtual goods for resale. In most lower income nation this activity offers higher wages than other jobs - and a the same time requires significantly less education and/or experience.

On the national level, expanding real money trading can have positive effects on the nation's trade balance. Because at the time all virtual worlds are recreational, subscribers with higher income are typically buyers in the real money trading transactions, whereas subscribers from low income nations tend to be sellers. This service export improves the trade balance, and also fuels internal growth: the new caste of virtual workers are capable of purchasing more goods and services, thus creating demand for more internally produced goods.

Real money trading has real dangers, too. On the one hand, it might lead to Dutch disease, thus distorting the exchange rate of the nation's currency. Left unattended, it can also lead to virtual colonization, where the real profits of real money trading would end up in the higher income nations. A way to fight against it is to help develop local vertical integrations by providing help to develop local trading houses. The government, however, would be hard pressed to directly regain this investment, since real money trading is largely invisible and leads to tax evasion.

\section{References}

1 Appadurai A, Modernity at large, University of Minnesota Press, Minneapolis, 1996.

2 Bruce S, January 2007, 10, available at wWw. mmorpgchart.com

3 Castronova E, Synthetic worlds, The University of Chicago Press, Chicago, 2005.

4 Hofer S (ed.), Prices and Earnings, Zurich, 2006. UBS AG.

5 Kelly R, Massively Multiplayer Online Role-Playing Games: The People, the Addiction and the Playing Experience, McFarland \& Company, New York, 2004.

6 Linden Labs (n.d.), Second Life Economic Statistics, March 17, 2007, available at $\mathrm{Ww}$. secondlife.com

7 Nkusu M, Aid and the Dutch Disease in Low-Income Countries: Informed Diagnoses for Prudent Prognoses, 2004. IMF working paper WP/04/49.

8 Proudfoot Consulting, 2006, available at http://www. proudfootconsulting.com/displayfile.asp?id=94443 Proudfoot Productivity Report.

9 Ricardo D, On the Principles of Political Economy and Taxation, 1817.

10 Smith A, An Inquiry into the Nature and Causes of the Wealth of Nations, 1776.

11 Taylor TL (ed.), Play Between Worlds: Exploring Online Game Culture, The MIT Press, Massachusetts, 2006.

12 Vajda G, Online játék éhbérért, 2005, February 9, available at http:// index.hu/tech/net/robot0209/

13 VSG: personal interview with the leader of the RMT trading house VSG. 